10

\title{
Spectral Peculiarities of Multilayer Cholesteric Wedge-Cell System with Dye-Doped Polymer Layer*
}

\author{
(C) H. Gharagulyan ${ }^{1}$, T.M. Sarukhanyan ${ }^{1}$, A.V. Ninoyan ${ }^{1}$, A.H. Gevorgyan ${ }^{2,}$, and R.B. Alaverdyan ${ }^{1}$ \\ ${ }^{1}$ Department of Physics, Yerevan State University, \\ 0025 Yerevan, Armenia \\ ${ }^{2}$ School of Natural Sciences, Far Eastern Federal University, \\ 690920 Vladivostok, Russia \\ e-mail: herminegharagulyan@ysu.am
}

Received May 17, 2019

Accepted May 17, 2019

Accepted June 23, 2020

Spectral properties of the three-layered wedge-cell system of two identical cholesteric layers with an isotropic defect (dye-doped polymer layer) between them were investigated experimentally and theoretically. It was shown that multiple defect modes can be observed in this kind of system.s photonic bandgap which widen the application range of mentioned above system such as low threshold lasing, multi-position trigger, multiwavelength filters, light shutters, etc. Supporting simulation was also provided showing an agreement between experimental results and theoretical calculations. The problem was solved by Ambartsumian.s layer addition modified method.

Keywords: cholesteric liquid crystals, chirality, photonic bandgap, defect modes, tuning, laser dye, polymer layer.

* Полный текст статьи опубликован в „Optics and Spectroscopy“ 2020 V. 128. N 10. 